\title{
A next-generation bifunctional photosensitizer with improved water-solubility for photodynamic therapy and diagnosis
}

\author{
Hirotada Nishie ${ }^{1}$, Hiromi Kataoka ${ }^{1}$, Shigenobu Yanoㄹ ${ }^{2}$ Jun-ichi Kikuchi², Noriyuki \\ Hayashi $^{1}$, Atsushi Narumi ${ }^{3}$, Akihiro Nomoto ${ }^{4}$, Eiji Kubota ${ }^{1}$, Takashi Joh ${ }^{1}$ \\ ${ }^{1}$ Departments of Gastroenterology and Metabolism, Nagoya City University Graduate School of Medical Sciences, Mizuho-cho, \\ Mizuho-ku, Nagoya 467-8601, Japan \\ ${ }^{2}$ Graduate School of Materials Science, Nara Institute of Science and Technology, Ikoma, Nara 630-0192, Japan \\ ${ }^{3}$ Department of Organic Materials Science, Graduate School of Organic Materials Science, Yamagata University, Yamagata, \\ Yonezawa 992-8510, Japan \\ ${ }^{4}$ Department of Applied Chemistry, Graduate School of Engineering, Osaka Prefecture University, Nakaku, Sakai, Osaka \\ 599-8531, Japan \\ Correspondence to: Hiromi Kataoka, email: hkataoka@med.nagoya-cu.ac.jp \\ Keywords: oligosaccharide-conjugated chlorin, Warburg effect, glycoconjugated chlorin, photodynamic therapy, photodynamic \\ diagnosis \\ Received: August 21, 2016 \\ Accepted: September 23, 2016 \\ Published: September 30, 2016
}

\section{ABSTRACT}

Photodynamic therapy (PDT) exploits light interactions and photosensitizers to induce cytotoxic reactive oxygen species. Photodynamic diagnosis (PDD) uses the phenomenon of photosensitizer emitting fluorescence to distinguish some tumors from normal tissue. The standard photosensitizer used for PDD is 5-aminolevulinic acid (5-ALA), although it is not entirely satisfactory. We previously reported glucoseconjugated chlorin (G-chlorin) as a more effective photosensitizer than another widely used photosensitizer, talaporfin sodium (TS); however, G-chlorin is hydrophobic. We synthesized oligosaccharide-conjugated chlorin (O-chlorin) with improved watersolubility. We report herein on its accumulation and cytotoxicity. O-chlorin was synthesized and examined for solubility. Flow cytometric analysis was performed to evaluate $\mathbf{0}$-chlorin accumulation in cancer cells. To evaluate the intracellular localization of photosensitizer, cells were stained with 0 -chlorin and organelle-specific fluorescent probes. We then measured the in vitro fluorescence of various photosensitizers and the half-maximal inhibitory concentrations to evaluate effects in PDD and PDT, respectively. Xenograft tumor models were established, and antitumor and visibility effects were analyzed. O-chlorin was first shown to be hydrophilic. Flow cytometry then revealed a 20- to 40-times higher accumulation of O-chlorin in cancer cells than of TS, and a 7- to 23-times greater fluorescence than 5-ALA. In vitro, the cytotoxicity of O-chlorin PDT was stronger than that of TS PDT, and O-chlorin tended to accumulate in lysosomes. In vivo, O-chlorin showed the best effect in PDT and PDD compared to other photosensitizers.

O-chlorin was hydrophilic and showed excellent tumor accumulation and fluorescence. $\mathbf{0}$-chlorin is promising as a next-generation bifunctional photosensitizer candidate for both PDT and PDD.

\section{INTRODUCTION}

Photosensitizers are molecules that undergo photochemical reactions in response to specific light irradiation to emit fluorescence. Photodynamic therapy (PDT) exploits this phenomenon to generate reactive oxygen species (ROS) such as singlet oxygen $[1,2]$, and is an established treatment for cancer and some nonmalignant diseases [2-6]. PDT has several advantage over conventional cancer treatment; it is relatively noninvasive and causes less systemic toxicity [3]. Furthermore, the same photosensitizers can also be used for photodynamic diagnosis (PDD) based on the emission of fluorescence when irradiated in the presence of specific abnormal cells. 
The antitumor mechanism of PDT manifests as three patterns. The first is a direct toxicity action due to ROS generation inside the tumor; the second is a shutdown effect induced by starving the surrounding vessels, leading to tumor infarction; and, the third is a normal immunological mechanism activated via the tumor response to PDT [1, 2, 7, 8]. The photosensitizer, 5-aminolevulinic acid (5ALA), which is converted at the tissue level to its active compound protoporphyrin IX, has been used historically for PDD in many medical fields because it accumulates strongly in tumor cells rather than in normal tissue due to their difference cellular metabolisms [9-13].

Since PDT and PDD were introduced more than a quarter of a century ago, various improvements have been attempted for better clinical results, including coordination of photochemical wavelengths, developments in laser generation, improved drug delivery systems, and of course, new and improved photosensitizer chemicals [14]. In this context, we previously reported a glucose-conjugated chlorin compound (G-chlorin) as a more effective photosensitizer than the conventional ones used thus far $[15,16]$, based on a phenomenon called the Warburg effects whereby cancer cells in general consume more glucose than normal cells [17]. Subsequently, we developed a mannose-conjugated chlorin (M-chlorin), which proved more efficient than G-chlorin in generating anti-tumor cytotoxicity and in suppressing tumor-associated macrophages [18]. However, when these glycoconjugated chlorins were administered intravenously, the reagents had to be dissolved into organic solvent due to their hydrophobicity. In recent years, Talaporfin sodium (TS), a second-generation photosensitizer in Japan, has been widely taken up for clinical use due to its improved water solubility and excretion [19, 20]; however, TS has not yet replaced the 'gold standard' for PDT. Furthermore, in the field of PDD, almost all reports cite 5-ALA because it specifically visualizes some tumors and has few side effects $[9,10,13$, 21, 22].

We recently succeeded in isolation of a novel glycoconjugated compound, hydrophilic O-chlorin having four maltotriose units [23], In this study we evaluated its cancer-selective accumulation, discrimination, and cytotoxicity as a bifunctional photosensitizer for both PDT and PDD.

\section{RESULTS}

\section{Newly synthesized O-chlorin showed water solubility}

We recently synthesized a glycoconjugated chlorin named O-chlorin that contains glucose-derived oligosaccharides, such as maltotriose. Compared to G-chlorin, O-chlorin proved to be more highly watersoluble (Figure 1E, 1F) [23]. This high solubility enabled

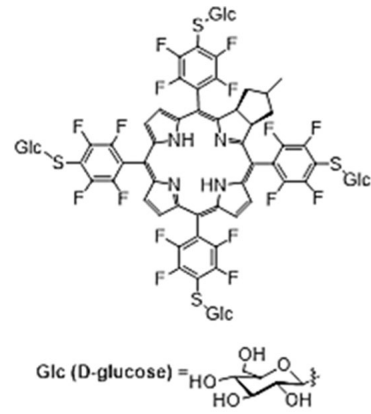

A

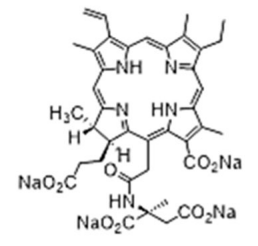

C

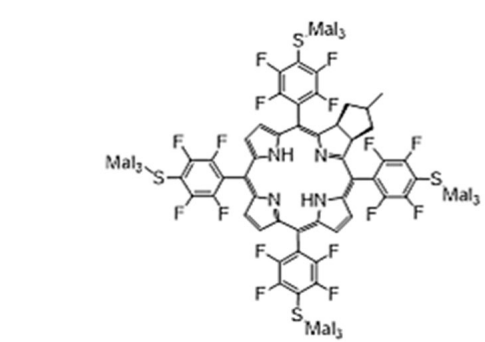

$\mathrm{Mal}_{3}$ (maltotriose) $=$

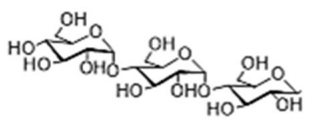

B

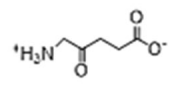

D

Figure 1: Chemical structure of G-chlorin, O-chlorin, TS, and 5-ALA and water solubility of O-chlorin. A. Glucoseconjugated chlorin (G-chlorin); 5, 10, 15, 20- tetrakis (4- ( $\beta$ - D- glucopyranosylthio)- 2, 3, 5, 6- tetrafluorophenyl)- 2, 3- (methano $(N$-methyl) iminomethano) chlorin. B. oligosaccharide(maltotrisose)-conjugated chlorin (O-chlorin); 5, 10, 15, 20- tetrakis (4- $(\beta$ - Dmaltotriosylthio)- 2, 3, 5, 6- tetrafluorophenyl)- 2, 3- (methano ( $N$-methyl) iminomethano) chlorin. C. talaporfin sodium ; (mono-l-aspartyl chlorin6) Laserphyrin ${ }^{\circledR}$. D. 5-ALA (5-Aminolevulinic acid). (A; G-chlorin, B: O-chlorin, C: TS, D: 5-ALA). G-chlorin solution was opaque due to its water insolubility, whereas O-chlorin was clear. (E. G-chlorin solution, F. O-chlorin solution). 
O-chlorin to be administered intravenously and to reach the whole body without addition of a toxic organic solvent.

\section{The accumulation of $\mathrm{O}$-chlorin in cancer cells was much higher than that of TS}

We first examined the uptake of TS and O-chlorin in vitro using MKN45 and HT29 cells. Cells were incubated with $5 \mu \mathrm{M}$ photosensitizer for 4 hours, and uptake was estimated by measuring the intensity of the characteristic red fluorescence at the single cell level using FACS. The accumulation of O-chlorin in both cancer cells was 20-40 times higher than that of TS (Figure 2).

\section{O-chlorin accumulated in cancer cells and was mainly localized in lysosomes}

We tested the accumulation and subcellular localization of O-chlorin by confocal microscopy using fluorescence probes to mark intracellular organelles. Cells were loaded with O-chlorin and incubated with MitoTracker Green, LysoTracker Green, NBD C6 ceramide Green, or ERTracker Green to label mitochondria, lysosomes, Golgi, or endoplasmic reticula, respectively. The detection of O-chlorin tended to coincide with LysoTracker, indicating accumulation in lysosomes (Figure 3).

\section{The fluorescent activity of $\mathrm{O}$-chlorin exceeded that of 5-ALA}

We next evaluated the PDD ability in vitro by measuring fluorescence of TS, O-chlorin, and 5-ALA. MKN45 cells were incubated with TS, O-chlorin, or
5-ALA, and then analyzed by microplate reader to measure the fluorescence of each reagent. The intensity of O-chlorin fluorescence was strongest, followed by 5-ALA, and then TS under the condition of $405 \mathrm{~nm}$ and $420 \mathrm{~nm}$ excitation and $635 \mathrm{~nm}$ and $650 \mathrm{~nm}$ emission (Figure 4). In various cancer cell lines, the fluorescence of O-chlorin was 7 to 23 times stronger than that of 5-ALA (Table 1).

\section{PDD with O-chlorin clearly identified tumors in vivo}

We then investigated the photosensitizer fluorescence in a xenograft tumor mice model. Once the implanted tumors reached a sufficient size, O-chlorin, 5-ALA, or TS were administered to mice at a dose of $2.5 \mu \mathrm{mol} / \mathrm{kg}, 50 \mu \mathrm{mol} / \mathrm{kg}$, or $2.5 \mu \mathrm{mol} / \mathrm{kg}$, respectively, and all mice were sacrificed 4 hours later. Tumor and various organs were then extracted and observed under white light and LED light irradiation. No difference was seen among the photosensitizers under white light; however, under $405 \mathrm{~nm}$ and $420 \mathrm{~nm}$ LED light, tumors administered with O-chlorin clearly emitted red fluorescence, those receiving TS or 5-ALA emitted little. Furthermore, there was little or no emission from the nontumor organs for all reagents (Figure 5).

\section{PDT with O-chlorin showed higher cytotoxicity than that with TS in esophageal, gastric, and colon cancer cells}

To evaluate the effectiveness of PDT with O-chlorin, we evaluated the cell death induced by PDT using O-chlorin. Cells were loaded with TS or O-chlorin for 24 hours, irradiated with 660-nm red LED light at $16 \mathrm{~J} / \mathrm{cm}^{2}$,

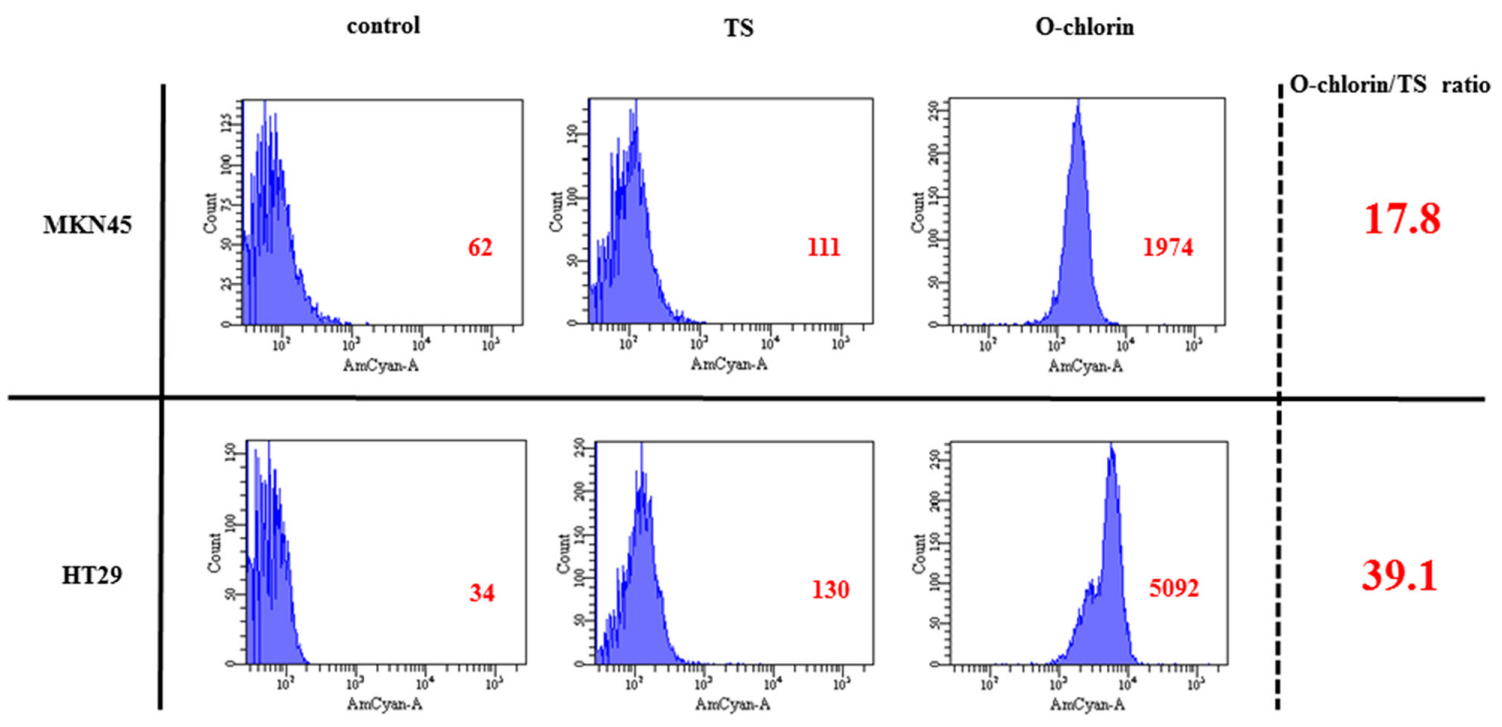

Figure 2: The accumulation of O-chlorin and TS in cancer cells. MKN45 and HT29 were loaded with O-chlorin, TS, or no reagent as a control for $4 \mathrm{~h}$, and then analyzed for cell accumulation using flow cytometry at $405 \mathrm{~nm}$ excitation and $680 \mathrm{~nm}$ emission. O-chlorin and TS both contained chlorin as a photosensitizer, with nearly peak excitation and emission wavelengths reached for the chlorin. The abscissa of the graph indicates populations of cells and the ordinate represents the intensity of emission. Red figures show mean areas. 
and incubated for 24 hours. We performed WST assays to determine the $\mathrm{IC}_{50}$ at 24 hours after irradiation. As shown in Table 2, PDT using O-chlorin induced cell death with 7 to 50 times higher cytotoxicity than TS in all cancer cells.

\section{O-chlorin PDT suppressed tumor growth strongly in vivo}

We finally evaluated the antitumor effects of O-chlorin PDT on xenograft tumors in mice. 14 days after tumor inoculation, mice were administered O-chlorin or TS intravenously at a dose of $0.625 \mu \mathrm{mol} / \mathrm{kg}$ or $6.25 \mu \mathrm{mol} /$ $\mathrm{kg}$, respectively. After 4 hours, tumors ware irradiated with the 664-nm LASAR at $15 \mathrm{~J} / \mathrm{cm}^{2}$. The PDT using O-chlorin strongly suppressed tumor growth compared to TS, even at one-tenth the amount of TS (Figure 6).

\section{DISCUSSION}

In this study we proved that newly developed O-chlorin PDT was superior to TS PDT, the secondgeneration PDT widely used in Japan, due to increased tumor accumulation and cytotoxicity. We previously reported that conjugating sugar chains onto photosensitizer compounds increases their cellular uptake and antitumor effect, thus glycoconjugated chlorin reagents should achieve more effective PDT than TS PDT. While G-chlorin and M-Chlorin proved to be water insoluble $[15,16,18]$, the recently synthesized O-chlorin has higher water-solubility by virtue of more attached sugar chains than G-chlorin (Figure 1E, 1F) [23]. For PDD, O-chlorin showed higher tumor accumulation than 5-ALA, the longheld 'gold standard' in clinical use worldwide for PDD.

With respect to the concentration of photosensitizer needed for PDT, Allison et al. [7] recommended using as little photosensitizer as possible to achieve a sufficient anti-tumor response. The higher cellular uptake and accumulation shown by O-chlorin in vitro and in vivo compared to TS should therefore enable lower amounts of photosensitizer to be used clinically for PDT. Such an outcome would also reduce associated adverse events like skin disorders. Indeed, in this study, the halfmaximal inhibitory concentration $\left(\mathrm{IC}_{50}\right)$ of O-chlorin in cancer cells was 11-59 times lower than that of TS. PDT

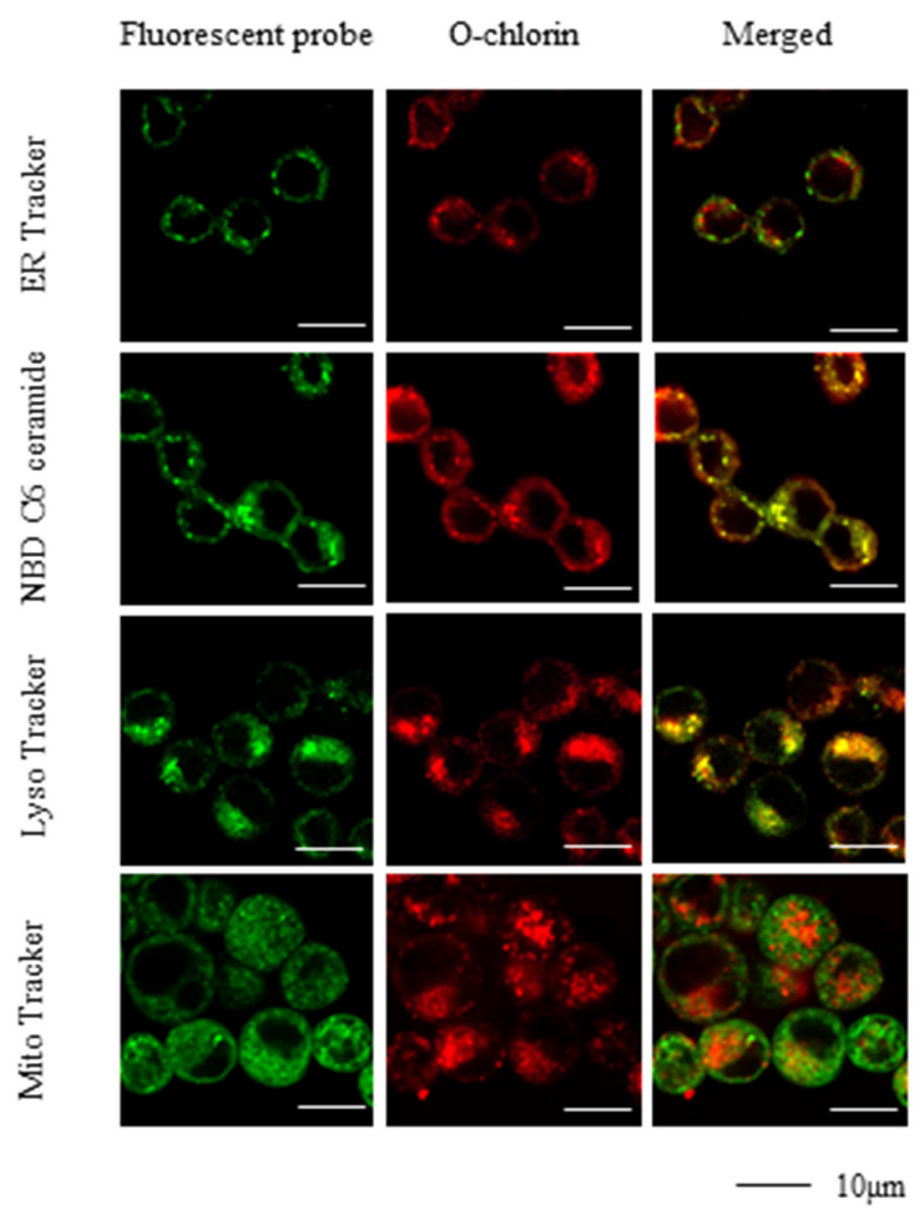

Figure 3: Subcellular localization of O-chlorin. MKN45 cells were loaded with O-chlorin for 24 hours and labeled with Mito Tracker Green, Lyso Tracker Green, NBD C6 ceramide Green, or ER Tracker Green. The images were obtained by confocal microscopy (Original magnification, $\times 300$; scale bar, $10 \mu \mathrm{m}$ ). 
effects on patients also vary according to the localization of photosensitizer accumulation. ROS generated via PDT have a short half-life and acts close to their site of generation. Consequently, the type of photodamage to cells loaded with a photosensitizer could depend on its precise subcellular localization [8]. Herein, we localized O-chlorin mainly in the lysosomes of cancer cells, at sites proposed to be critical for photosensitizer action [24]. However, later studies reported that although lysosomally localized photosensitizers can lead to cell killing upon illumination, the relative efficacy is significantly lower than that seen with photosensitizers localized in mitochondria and other organelles [25]. Mitochondrial damage after illumination is a particularly important mechanism of apoptotic cell death induced via PDT [26]. Nagata et al. [27] also showed lysosomes as the primary site of chlorin-

\section{EX405nm/Em635nm}

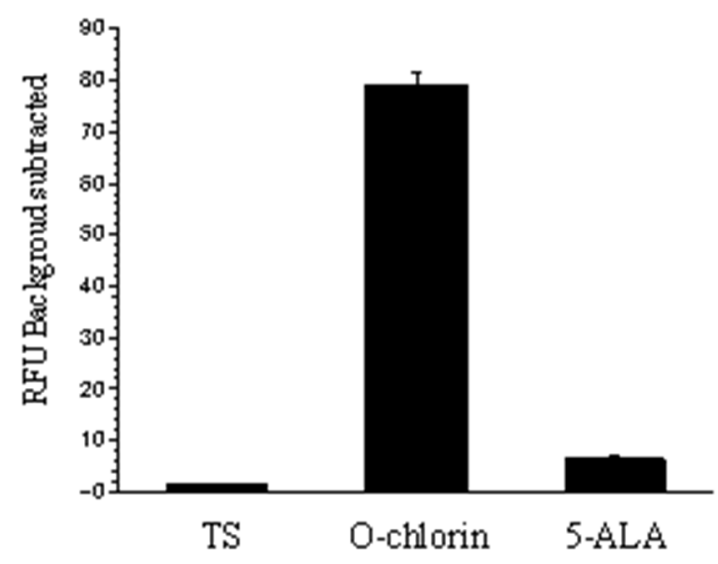

$\mathrm{EX} 420 \mathrm{~nm} / \mathrm{Em} 635 \mathrm{~nm}$

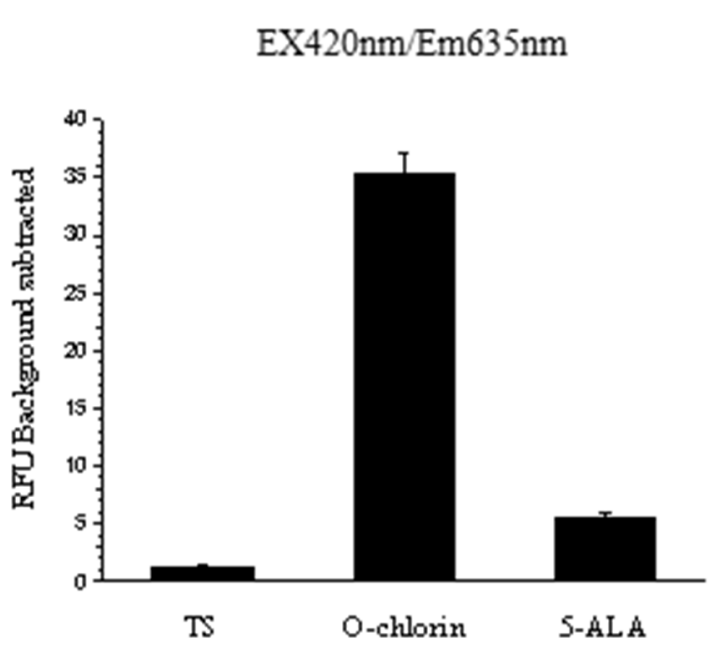

based photosensitizer accumulation, and that these cells underwent apoptosis upon irradiation doses leading to $70 \%$ cell death, suggesting that apoptotic pathways are activated via mitochondrial destabilization following PDT damage to lysosomes. Thus, the preferential accumulation of O-chlorin in cancer cell lysosomes in our studies could have induced apoptosis via lysosomal damage even with low doses of photosensitizer.

The characteristics of an ideal photosensitizer have been discussed in recent reviews and indicated important factors, including good water-solubility [28, 29]. O-chlorin would therefore be an ideal photosensitizer, with almost the same antitumor effect as G-chlorin and the same hydrophilicity as TS. For PDD, O-chlorin showed higher fluorescence activity in various cancer cell lines in vitro than 5-ALA, conventionally used

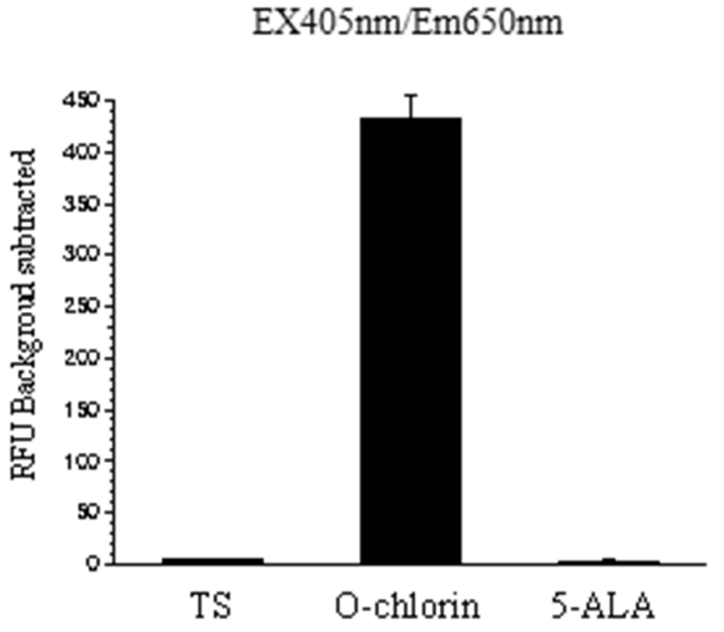

Figure 4: Fluorescence of photosensitizers in MKN45 cells under various irradiations of LED light. MKN45 cells were incubated with TS, O-chlorin, or 5-ALA, then fluorescence intensities were measured using a microplate reader. Light was irradiated under condition of $405 \mathrm{~nm}$ and $420 \mathrm{~nm}$ excitation, and $635 \mathrm{~nm}$ and $650 \mathrm{~nm}$ emission. Data are means $\pm \mathrm{SE}$ of eight independent experiments. 
Table 1: The effect of PDD among TS, 5-ALA and O-chlorin in various cell lines

\begin{tabular}{lcccc}
\hline \multicolumn{5}{c}{ Photosensitizer } \\
\hline Cell line & TS & 5-ALA & O-chlorin & O-chlorin/5-ALA ratio \\
\hline MKN45 & $1.97 \pm 0.20$ & $11.38 \pm 1.93$ & $80.26 \pm 2.77$ & 7.05 \\
HT29 & $1.52 \pm 0.10$ & $6.31 \pm 0.67$ & $79.17 \pm 2.40$ & 12.55 \\
OE21 & $2.39 \pm 0.11$ & $8.04 \pm 0.55$ & $76.78 \pm 3.40$ & 9.55 \\
KYSE30 & $2.92 \pm 0.14$ & $5.26 \pm 0.41$ & $123.09 \pm 2.54$ & 23.40 \\
\hline
\end{tabular}

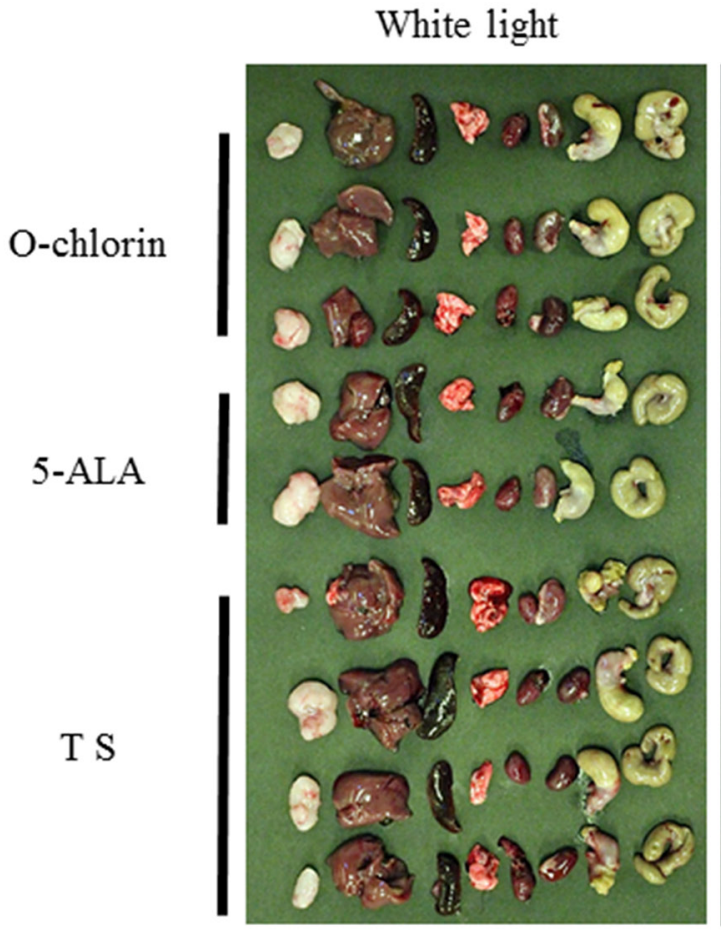

$\begin{array}{llllllll}1 & 2 & 3 & 4 & 5 & 6 & 7 & 8\end{array}$
$420 \mathrm{~nm}$

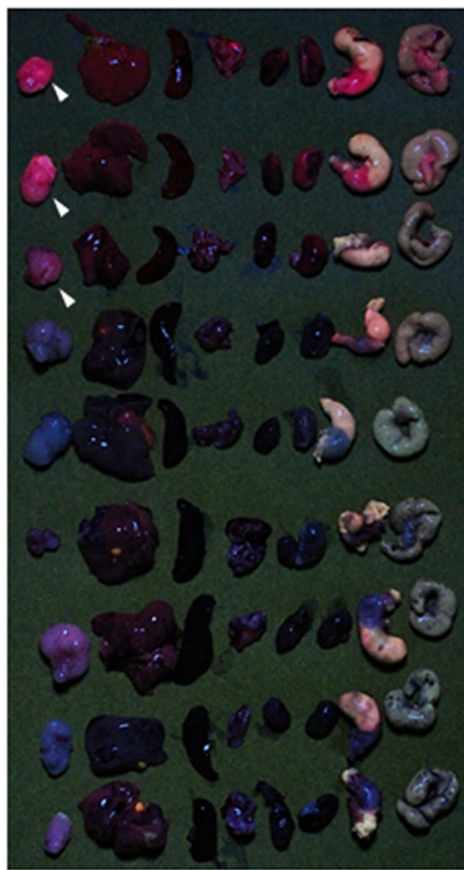

$\begin{array}{llllllll}1 & 2 & 3 & 4 & 5 & 6 & 7 & 8\end{array}$
$405 \mathrm{~nm}$

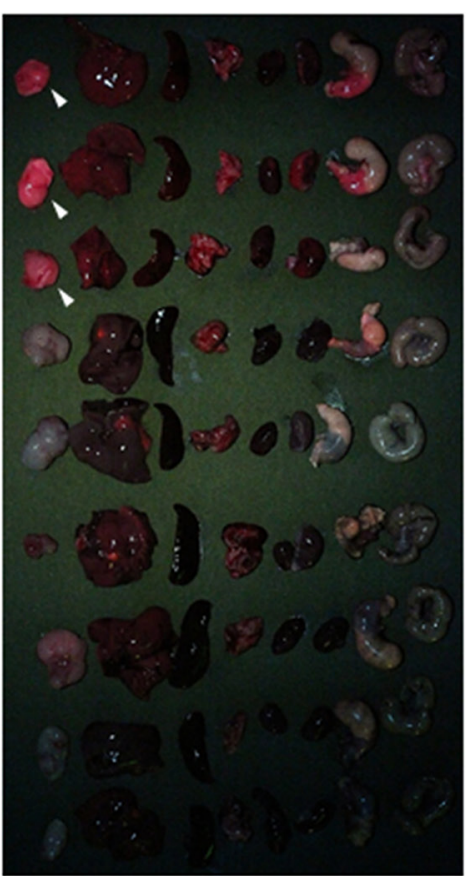

$\begin{array}{llllllll}1 & 2 & 3 & 4 & 5 & 6 & 7 & 8\end{array}$

Figure 5: PDD effect in xenograft tumor model. Once tumors reached a sufficient size, mice were administered O-chlorin, 5-ALA, or TS at a dose of $2.5 \mu \mathrm{mol} / \mathrm{kg}, 50 \mu \mathrm{mol} / \mathrm{kg}$, or $2.5 \mu \mathrm{mol} / \mathrm{kg}$, respectively. 4 hours after administration, mice were sacrificed, and tumor and organs were extracted. The excised tissues were observed under irradiation by white light and LED light of $405 \mathrm{~nm}$ and $420 \mathrm{~nm}$. The white arrowhead indicates tumors administered with O-chlorin and showing strong fluorescence. Sample number of O-chlorin, 5-ALA, and TS were three, two, and four, respectively. Numeral below the figure indicates tumor and organs (1; tumor, 2; liver, 3; spleen, 4; lung, 5; heart, 6; kidney, 7; stomach, 8; colon).

Table 2: Comparison of 50\% inhibition concentration $\left(\mathrm{IC}_{50}\right)$ by PDT between TS and O-chlorin

\begin{tabular}{lccccc}
\hline & \multicolumn{2}{c}{ Esophageal cancer } & \multicolumn{2}{c}{ Gastric cancer } & \multicolumn{2}{c}{ Colon cancer } \\
\cline { 2 - 6 } & OE21 & KYSE30 & MKN28 & MKN45 & HT29 \\
\hline TS & 17.4 & 4.29 & 12.7 & 11.4 & 18.55 \\
O-chlorin & 0.33 & 0.11 & 0.56 & 1.25 & 0.97 \\
\hline
\end{tabular}




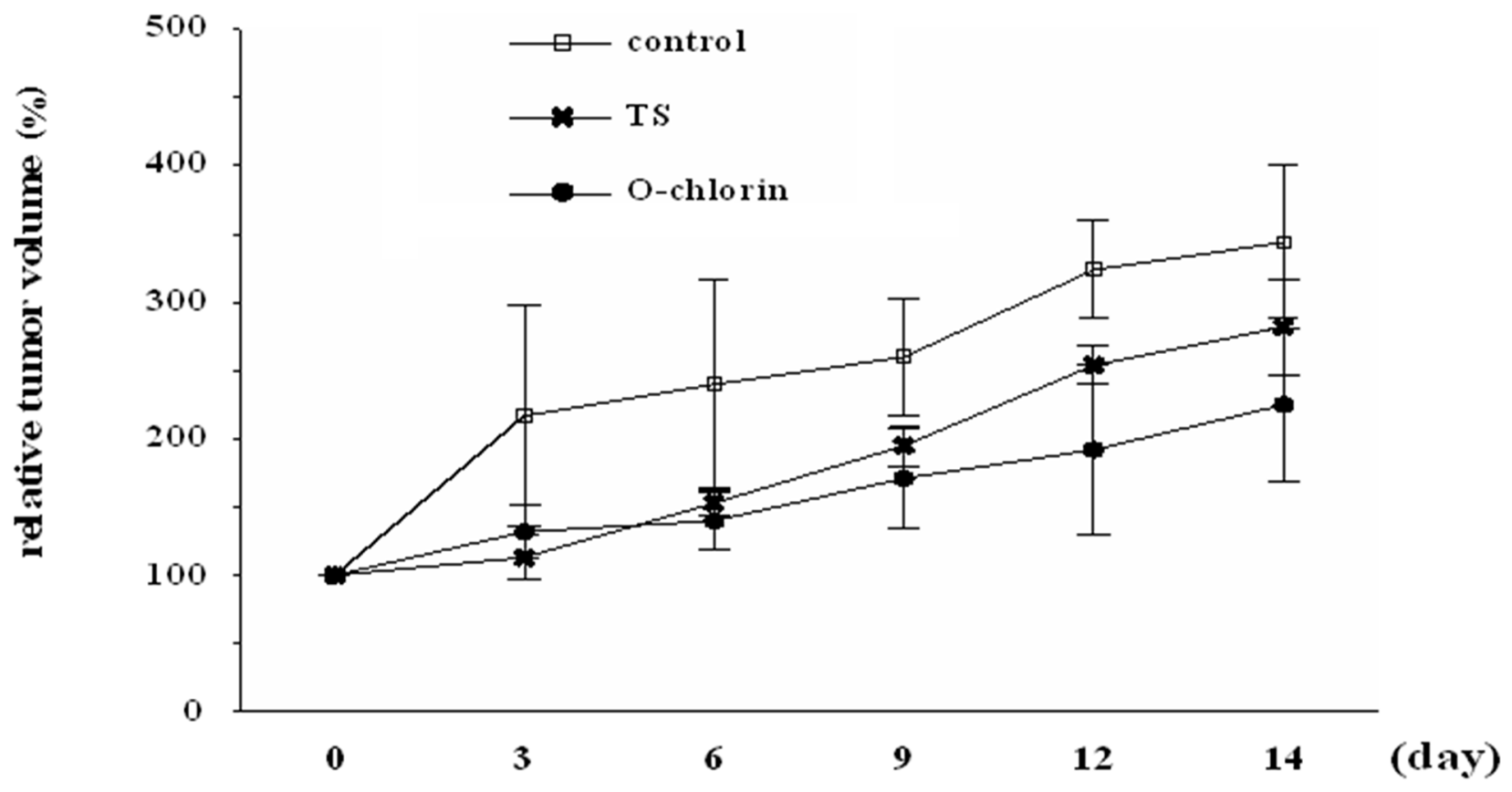

Figure 6: Antitumor effects of PDT in mouse xenograft model. Mice were irradiated with $15 \mathrm{~J} / \mathrm{cm}^{2}$ of diode laser at $664 \mathrm{~nm}$ 4 hours after injection of the photosensitizer. PDT was performed on day 0 and tumor volumes were monitored for 14 days in total. Data are shown as $\pm \mathrm{SE}$ ( $\mathrm{n}=3$ for control, $\mathrm{n}=6$ for TS and O-chlorin).

worldwide. Moreover, the xenograft tumors dosed with O-chlorin clearly emitted fluorescence in vivo, while those with TS and 5-ALA emitted little (Figure 4). For over 25 years, PDD using 5-ALA was used in various fields such as neurosurgery, urology, gastroenterology, and gynecology. Especially in neurosurgery and urology, PDD has an increasingly important role in deciding surgical margins prior to and during surgery, resulting in improved prognoses [9-13, 30, 31]. Based on this study, O-chlorin has prospective value as a novel and conventional PDD reagent, to detect and discriminate various tumor locations.

In terms of mechanism, we speculate that the major antitumor effect of O-chlorin PDT could be ROSinduced direct toxicity, as for TS. PDT using TS also works by tumor shutdown, and our in vivo examinations implicated this mechanism for O-chlorin PDT because TS and O-chlorin have the same chemical structure. The precise mechanism of O-chlorin PDT remains to be clarified.

In conclusion, we synthesized O-chlorin, which showed good water-solubility, strong accumulation to tumors, and high antitumor efficacy, suggesting it to be an efficient and tolerable reagent for PDT and PDD. Finally, O-chlorin has temporal and economic benefits by enabling PDT and PDD to be performed using a single photosensitizer and at the same time. Therefore, O-chlorin is a novel candidate for the ideal next-generation "bifunctional" photosensitizer.

\section{MATERIALS AND METHODS}

\section{Photosensitizers}

The photosensitizer compounds, O-chlorin (TFPC$\left.\mathrm{SMal}_{3}\right) \quad 5,10,15,20$-tetrakis-[4-( $\beta$-D-maltotriosylthio)2,3,5,6-tetrafluorophenyl]-2,3-[methano- (N-methyl) iminomethano]chlorin and G-chlorin ( $\mathrm{H}_{2}$ TFPC-SGlc) (5,10,15,20-tetrakis(pentafluorophenyl)-2,3-(methano[Nmethyl]iminomethano])chlorin) were synthesized and provided by laboratories at Yamagata University (Japan) and Nara Institute of Science and Technology (Japan) (Figure 1A, 1B). TS (mono-l-aspartyl chlorin6, Laserphyrin ${ }^{\circledR}$ ) was purchased from Meiji Seika (Tokyo, Japan) (Figure 1C), and 5-ALA was purchased from Cosmo Bio Co., LTD (Tokyo, Japan) (Figure 1D).

\section{Cell culture}

The human esophageal cancer cell line OE21 (No.11D028; ECACC) was cultured in RPMI 1640 medium (Wako Pure Chemical Industries) while KYSE30 was grown in a 50/50 media mix of RPMI 1640 and Ham's F12 (Wako Pure Chemical Industries) supplemented with $2 \mathrm{mM}$ glutamine, $2 \%$ fetal bovine serum (FBS), $100 \mathrm{U} / \mathrm{mL}$ penicillin, $100 \mathrm{mg} / \mathrm{mL}$ streptomycin, and $0.25 \mathrm{mg} / \mathrm{mL}$ amphotericin B. The HT29 colon cancer cell line (No. HTB-38; ATCC) was cultured in McCoy's 5A medium (Life Technologies) 
supplemented with $10 \%$ FBS and $100 \mathrm{U} / \mathrm{mL}$ penicillin, $100 \mathrm{mg} / \mathrm{mL}$ streptomycin, and $0.25 \mathrm{mg} / \mathrm{mL}$ amphotericin B. The MKN45 human gastric cancer cell line (No. 0254; Japanese Cancer Research Bank) was cultured in RPMI 1640 supplemented with 10\% FBS and $100 \mathrm{U} / \mathrm{mL}$ penicillin, $100 \mathrm{mg} / \mathrm{mL}$ streptomycin, and $0.25 \mathrm{mg} / \mathrm{mL}$ amphotericin B. Cells were cultured under an atmosphere of $5 \% \mathrm{CO}_{2}$ at $37^{\circ} \mathrm{C}$.

\section{Animals and tumor models}

Female nude mice (BALB/c Slc-nu/nu) of 4-6 weeks old and weighing15-20 g were purchased from Japan SLC. Before initiating any interventions, mice were allowed to acclimatize for at least 2 weeks in the animal facility. Xenograft tumor models were established by subcutaneously implanting $1 \times 10^{6}$ MKN45 cells in 100 $\mathrm{mL}$ of culture media under the right flank of experimental mice. The procedures and experiments were approved by Nagoya City University Center for Experimental Animal Science, and mice were cared for according to the guidelines of the Nagoya City University for Animal Experiments.

\section{Flow cytometric analysis}

Cancer cells were seeded into $6-\mathrm{cm}$ culture dishes at $2 \times 10^{5}$ cells/well and incubated at $37^{\circ} \mathrm{C}$ for 48 hours. After removing the medium to evaluate the accumulation of photosensitizer into cells, fresh medium supplemented with $5 \mu \mathrm{M}$ photosensitizer were added to the dishes for 4 hours. Cells were then washed with phosphate-buffered saline (PBS) three times and removed from the culture dish with TrypLE-Express (Invitrogen) for analysis using a FACSCant II (BD Biosciences) at excitation and emission wavelengths of $405 \mathrm{~nm}$ and $680 \mathrm{~nm}$, respectively. At least 10,000 events were collected for each sample.

\section{Intracellular localization of photosensitizers}

MKN45 cells were seeded onto coverslips placed in 12 -well culture plates at $1 \times 10^{5}$ cells/well and incubated for 24 hours. Subsequently, $1 \mu \mathrm{mol} / \mathrm{L}$ O-chlorin was added to the culture media and the cells were incubated for a further 4 hours before staining with organellespecific fluorescent probes. Lysosomes were stained with $0.1 \mu \mathrm{mol} / \mathrm{L}$ LysoTracker Green (Invitrogen) at $37^{\circ} \mathrm{C}$ for 30 minutes, mitochondria with $0.1 \mu \mathrm{mol} / \mathrm{L}$ MitoTracker Green FM (Invitrogen) at $37^{\circ} \mathrm{C}$ for 10 minutes, Golgi with $5 \mu \mathrm{mol} / \mathrm{L}$ NBD C6-ceramide at $4{ }^{\circ} \mathrm{C}$ for 30 minutes, and endoplasmic reticulum with $0.1 \mu \mathrm{mol} / \mathrm{L}$ ER-Tracker Green (Invitrogen) at $37^{\circ} \mathrm{C}$ for 30 minutes. After incubation, culture media were replaced with fresh medium to remove free dyes, and then the stained cells for observed, live for mitochondria and following fixation with 4\% paraformaldehyde for lysosomes, Golgi, and endoplasmic reticulum. To visualize the localization, confocal laser microscopy (Nikon A1 confocal system Nikon Instech Co., Ltd.) was used and the obtained data were analyzed with NIS element imaging software (Nikon). Band-pass emission filters of 505-530 nm and $650 \mathrm{~nm}$ were used.

\section{Fluorescence of photosensitizers in vitro}

The effect of PDD in vitro was evaluated by measuring fluorescence in the cancer cell lines. The esophageal, gastric, and colon cancer cells (OE21, KYSE30, MKN45, HT29) were seeded in opaque 96well culture plates at $5 \times 10^{3}$ cells $/ 100 \mathrm{~mL} /$ well and incubated for 24 hours. The media were removed and the cells were incubated for a further 4 hours in medium supplemented with TS, 5-ALA, or O-chlorin at $5 \mu \mathrm{mol} / \mathrm{L}, 1 \mathrm{mmol} / \mathrm{L}$, and $5 \mu \mathrm{mol} / \mathrm{L}$, respectively. Then, cells were washed three times with PBS and covered left in PBS until the fluorescence of each reagent was measured using a microplate reader (Gemini EM, Molecular Devices). Intensity of fluorescence was expressed as a relative fluorescence unit background subtracted. The obtained data were analyzed with SoftMAX pro software (Molecular Devices). The wavelengths of excitation used for analysis were 405 $\mathrm{nm}$ and $420 \mathrm{~nm}$, and for emission were $635 \mathrm{~nm}$ and $650 \mathrm{~nm}$, with data collected from eight independent experiments.

\section{In vivo PDD}

After the tumor implanted on mice reached approximately $100 \mathrm{~mm}^{3}$, solution of TS, 5-ALA, or O-chlorin was injected via the tail vein at a dose of $2.5 \mu \mathrm{mol} / \mathrm{kg}, 50 \mu \mathrm{mol} / \mathrm{kg}$ and $2.5 \mu \mathrm{mol} / \mathrm{kg}$, respectively. After 4 hours, mice were sacrificed according to our institutional guidelines followed by extraction of tumor and organs including liver, spleen, lung, heart, kidney, stomach, and colon. Subsequently, tumor and organs were observed under white light and under $405 \mathrm{~nm}$ and $420 \mathrm{~nm}$ irradiation of LED light. The image was obtained using a high resolution camera equipped with optical filter (cuton:470 nm, Longpass Filter/VIS 470nm, Asahi Spectra Co., Ltd).

\section{In vitro $\mathrm{PDT}$}

The esophageal, gastric, and colon cancer cells (OE21, KYSE30, MKN45, HT29) were incubated with photosensitizer in culture medium. After 24 hours, cancer cells were washed once with PBS, covered with PBS, and irradiated with LED light (Optocode corporation) which emits $660 \mathrm{~nm}$ wavelength at an energy of $16 \mathrm{~J} / \mathrm{cm}^{2}$ (intensity: $36 \mathrm{~mW} / \mathrm{cm}^{2}$ ). 


\section{Cell viability assay}

Cell viability was evaluated by the WST- 8 cell proliferation assay (Dojindo). Cancer cells were seeded in 96 -well culture plates at $5 \times 10^{3}$ cells $/ 100 \mathrm{~mL} /$ well and incubated overnight. Subsequently, cells were incubated with TS or O-chlorin at $37^{\circ} \mathrm{C}$ for 24 hours, irradiated, and then incubated with culture medium for a further 24 hours. Cells were incubated with the cell counting kit- 8 solution for 2 hours and absorption at $450 \mathrm{~nm}$ was measured using a microplate reader (SPECTRA MAX340, Molecular Devices). Cell viability was expressed as a percentage of untreated control cells. The half-maximal (50\%) inhibitory concentration $\left(\mathrm{IC}_{50}\right)$ was calculated for each reagent.

\section{In vivo PDT}

When the implanted tumors grew to approximately $100 \mathrm{~mm}^{3}$, mice were divided into three groups with comparable average tumor volumes. Subsequently, O-chlorin or TS was administered to mice in the appropriate groups via tail vein injection at a dose of 0.625 $\mu \mathrm{mol} / \mathrm{kg}$ or $6.25 \mu \mathrm{mol} / \mathrm{kg}$, respectively. Four hours after administration, the tumors were irradiated using a 664$\mathrm{nm}$ red LASER (OK Fiber Technology) at a dose of $15 \mathrm{~J} /$ $\mathrm{cm}^{2}$ (intensity: $150 \mathrm{~mW} / \mathrm{cm}^{2}$ ) applied to the skin directly above the tumors. Treatment was performed only once at 7 to10 days after tumor inoculation. Tumor growth was monitored once every three days by measuring the tumor volume with Vernier calipers and the tumor volume was calculated by the formula, length $\times$ width $\times \operatorname{depth} / 2$. The results were analyzed using the Bonferroni-Holm method to assess differences between groups.

\section{ACKNOWLEDGMENTS}

We are grateful to Ikumi Nomura and Yukimi Ito at Nagoya City University Graduate School of Medical Sciences for technical assistance. The authors acknowledge Yuichi Sakai of Asahi Kasei Chemicals Corporation for his useful support.

\section{CONFLICTS OF INTEREST}

The authors state that they have no potential conflicts of interest to disclose.

\section{GRANT SUPPORT}

This study was partially supported by JSPS KAKENHI grant numbers 26460947, 19350031, 25288028, the Japan-German Exchange Program supported by the JSPS and the Deutsche Forschungsgemeinschaft (DFG) and Translational Research Network Program from Japan Agency for Medical Research and Development, AMED, 2015-2016.

\section{REFERENCES}

1. Dolmans DE, Fukumura D and Jain RK. Photodynamic therapy for cancer. Nat Rev Cancer. 2003; 3:380-387.

2. Dougherty TJ, Gomer CJ, Henderson BW, Jori G, Kessel D, Korbelik M, Moan J and Peng Q. Photodynamic therapy. J Natl Cancer Inst. 1998; 90:889-905.

3. Brown SB, Brown EA and Walker I. The present and future role of photodynamic therapy in cancer treatment. Lancet Oncol. 2004; 5:497-508.

4. Petersen BT, Chuttani R, Croffie J, DiSario J, Liu J, Mishkin D, Shah R, Somogyi L, Tierney W and Wong Kee Song LM. Photodynamic therapy for gastrointestinal disease. Gastrointest Endosc. 2006; 63:927-932.

5. Yano S, Hirohara S, Obata M, Hagiya Y, Ogura S-i, Ikeda A, Kataoka H, Tanaka M and Joh T. Current states and future views in photodynamic therapy. Journal of Photochemistry and Photobiology C: Photochemistry Reviews. 2011; 12:46-67.

6. Huang Z. A Review of Progress in Clinical Photodynamic Therapy. Technol Cancer Res Treat. 2005; 4:283-293.

7. Allison RR and Moghissi K. Photodynamic Therapy (PDT): PDT Mechanisms. Clinical endoscopy. 2013; 46:24-29.

8. Castano AP, Demidova TN and Hamblin MR. Mechanisms in photodynamic therapy: part one-photosensitizers, photochemistry and cellular localization. Photodiagnosis Photodyn Ther. 2004; 1:279-293.

9. Stummer W, Pichlmeier U, Meinel T, Wiestler OD, Zanella $\mathrm{F}$ and Reulen HJ. Fluorescence-guided surgery with 5-aminolevulinic acid for resection of malignant glioma: a randomised controlled multicentre phase III trial. Lancet Oncol. 2006; 7:392-401.

10. Motoori M, Yano M, Tanaka K, Kishi K, Takahashi H, Inoue M, Saito T, Sugimura K, Fujiwara Y, Ishikawa O and Sakon M. Intraoperative photodynamic diagnosis of lymph node metastasis in esophageal cancer patients using 5-aminolevulinic acid. Oncol Lett. 2015; 10:3035-3039.

11. Kondo Y, Murayama Y, Konishi H, Morimura R, Komatsu S, Shiozaki A, Kuriu Y, Ikoma H, Kubota T, Nakanishi M, Ichikawa D, Fujiwara H, Okamoto K, et al. Fluorescent detection of peritoneal metastasis in human colorectal cancer using 5-aminolevulinic acid. Int J Oncol. 2014; 45:41-46.

12. Hinnen P, de Rooij FW, van Velthuysen ML, Edixhoven A, van Hillegersberg R, Tilanus HW, Wilson JH and Siersema PD. Biochemical basis of 5-aminolaevulinic acid-induced protoporphyrin IX accumulation: a study in patients with (pre)malignant lesions of the oesophagus. Br J Cancer. 1998; 78:679-682.

13. Denzinger S, Burger M, Walter B, Knuechel R, Roessler W, Wieland WF and Filbeck T. Clinically relevant reduction in risk of recurrence of superficial bladder cancer using 5-aminolevulinic acid-induced fluorescence diagnosis: 
8 -year results of prospective randomized study. Urology. 2007; 69:675-679.

14. Juarranz A, Jaen P, Sanz-Rodriguez F, Cuevas J and Gonzalez S. Photodynamic therapy of cancer. Basic principles and applications. Clin Transl Oncol. 2008; 10:148-154.

15. Tanaka M, Kataoka H, Mabuchi M, Sakuma S, Takahashi S, Tujii R, Akashi H, Ohi H, Yano S, Morita A and Joh T. Anticancer effects of novel photodynamic therapy with glycoconjugated chlorin for gastric and colon cancer. Anticancer Res. 2011; 31:763-769.

16. Tanaka M, Kataoka H, Yano S, Ohi H, Moriwaki K, Akashi H, Taguchi T, Hayashi N, Hamano S, Mori Y, Kubota E, Tanida S and Joh T. Antitumor effects in gastrointestinal stromal tumors using photodynamic therapy with a novel glucose-conjugated chlorin. Mol Cancer Ther. 2014; 13:767-775.

17. Warburg O. On the origin of cancer cells. Science. 1956; 123:309-314.

18. Hayashi N, Kataoka H, Yano S, Tanaka M, Moriwaki K, Akashi H, Suzuki S, Mori Y, Kubota E, Tanida S, Takahashi $\mathrm{S}$ and Joh T. A novel photodynamic therapy targeting cancer cells and tumor-associated macrophages. Mol Cancer Ther. $2015 ; 14: 452-460$.

19. Miki Y, Akimoto J, Hiranuma M and Fujiwara Y. Effect of talaporfin sodium-mediated photodynamic therapy on cell death modalities in human glioblastoma T98G cells. J Toxicol Sci. 2014; 39:821-827.

20. Tsutsumi M, Miki Y, Akimoto J, Haraoka J, Aizawa K, Hirano K and Beppu M. Photodynamic therapy with talaporfin sodium induces dose-dependent apoptotic cell death in human glioma cell lines. Photodiagnosis Photodyn Ther. 2013; 10:103-110.

21. Namikawa T, Yatabe T, Inoue K, Shuin T and Hanazaki K. Clinical applications of 5-aminolevulinic acid-mediated fluorescence for gastric cancer. World J Gastroenterol. 2015; 21:8769-8775.

22. Regula J, MacRobert AJ, Gorchein A, Buonaccorsi GA, Thorpe SM, Spencer GM, Hatfield AR and Bown SG. Photosensitisation and photodynamic therapy of oesophageal, duodenal, and colorectal tumours using 5 aminolaevulinic acid induced protoporphyrin IX--a pilot study. Gut. 1995; 36:67-75.
23. Narumi A, Tsuji T, Shinohara K, Yamazaki H, Kikuchi M, Kawaguchi S, Mae T, Ikeda A, Sakai Y, Kataoka H, Inoue A, Nomoto A, Kikuchi J-i and Yano S. MaltotrioseConjugation to Fluorinated Chlorin Derivative Generating PDT Photosensitizer with Improved Water-Solubility. Org Biomol Chem. 2016.

24. Geze M, Morliere P, Maziere JC, Smith KM and Santus R. Lysosomes, a key target of hydrophobic photosensitizers proposed for photochemotherapeutic applications. J Photochem Photobiol B. 1993; 20:23-35.

25. MacDonald IJ, Morgan J, Bellnier DA, Paszkiewicz GM, Whitaker JE, Litchfield DJ and Dougherty TJ. Subcellular localization patterns and their relationship to photodynamic activity of pyropheophorbide-a derivatives. Photochem Photobiol. 1999; 70:789-797.

26. Morgan J and Oseroff AR. Mitochondria-based photodynamic anti-cancer therapy. Advanced drug delivery reviews. 2001; 49:71-86.

27. Nagata S, Obana A, Gohto Y and Nakajima S. Necrotic and apoptotic cell death of human malignant melanoma cells following photodynamic therapy using an amphiphilic photosensitizer, ATX-S10(Na). Lasers Surg Med. 2003; 33:64-70.

28. Detty MR, Gibson SL and Wagner SJ. Current clinical and preclinical photosensitizers for use in photodynamic therapy. J Med Chem. 2004; 47:3897-3915.

29. Allison RR, Downie GH, Cuenca R, Hu XH, Childs CJ and Sibata CH. Photosensitizers in clinical PDT. Photodiagnosis Photodyn Ther. 2004; 1:27-42.

30. Roberts DW, Valdes PA, Harris BT, Fontaine KM, Hartov A, Fan X, Ji S, Lollis SS, Pogue BW, Leblond F, Tosteson TD, Wilson BC and Paulsen KD. Coregistered fluorescenceenhanced tumor resection of malignant glioma: relationships between delta-aminolevulinic acid-induced protoporphyrin IX fluorescence, magnetic resonance imaging enhancement, and neuropathological parameters. Clinical article. J Neurosurg. 2011; 114:595-603.

31. Babjuk M, Soukup V, Petrik R, Jirsa M and Dvoracek J. 5-aminolaevulinic acid-induced fluorescence cystoscopy during transurethral resection reduces the risk of recurrence in stage $\mathrm{Ta} / \mathrm{T} 1$ bladder cancer. BJU Int. 2005; 96:798-802. 